\title{
Management of Simultaneous Biliary and Duodenal Obstruction: The Endoscopic Perspective
}

\author{
Todd H. Baron \\ Division of Gastroenterology and Hepatology, Department of Medicine, Mayo Clinic, Rochester, MN, USA
}

Obstructive jaundice often develops in patients with unresectable malignancy in and around the head of the pancreas. Duodenal obstruction can also occur in these patients, and usually develops late in the disease course. Palliation of both malignant biliary and duodenal obstruction is traditionally performed with surgical diversion of the bile duct and stomach, respectively. With the advent of nonsurgical palliation of biliary obstruction using endoscopic transpapillary expandable metal stent placement, a similar approach can be used to palliate duodenal obstruction by placement of expandable metal gastroduodenal stents. Endoscopic palliation can be achieved in patients who require relief of both biliary obstruction and duodenal obstruction, although this can be technically difficult to achieve depending on the level of duodenal obstruction in relation to the major papilla. This article reviews the endoscopic approaches for combined palliative relief of malignant biliary and duodenal obstruction. (Gut Liver 2010;4(Suppl. 1):S50-56)

Key Words: Duodenal obstruction; Endoscopy, gastrointestinal; Palliative care; Stents; Cholestasis

\section{INTRODUCTION}

Patients with pancreatic and peri-pancreatic cancer (including, ampullary cancer, cholangiocarcinoma, gallbladder cancer and metastatic lesions in and around the head of pancreas) are usually diagnosed at an advanced stage in which curative resection is impossible. The median survival rate of patients with non-resectable peri-pancreatic cancer varies widely and can be as long as 6 to 12 months. However, in the setting of both biliary and duo- denal obstruction the survival time is shorter. ${ }^{1}$ During this period treatment should focus on palliation of symptoms of obstructive jaundice, duodenal obstruction and pain. Controversy exists about how to provide the optimal palliative treatment. Both surgical and non-surgical palliative procedures can be used to relieve obstructive jaundice and duodenal obstruction. Surgical palliation is achieved by biliary and gastric diversion through the creation of choledochojejunal and gastrojejunal anastomoses, respectively.

Endoscopic placement of self-expandable metal stents (SEMS) has been used to palliate biliary ${ }^{2}$ and duodenal obstruction. ${ }^{3}$ It is known that many patients who require duodenal stent placement also have undergone or will require subsequent palliation of biliary obstruction. ${ }^{4}$ In this article the endoscopic methods used for combined relief of both malignant biliary and duodenal obstruction will be reviewed.

\section{ANATOMIC AND CLINICAL SCENARIOS}

Anatomic and clinical scenarios determine the endoscopic approach to the palliative management of patients with both biliary and duodenal obstruction and will be discussed separately.

\section{Anatomic scenarios of biliary and duodenal ob- struction}

The location of the duodenal obstruction in relation to the major papilla is the major determinant to successful endoscopic simultaneous palliation of biliary and duodenal obstruction since the duodenal obstruction can limit access to the biliary opening. Mutignani et al. ${ }^{1}$ proposed a

\footnotetext{
Correspondence to: Todd H. Baron

Division of Gastroenterology and Hepatology, Department of Medicine, 200 First St. SW, Rochester, MN 55905, USA

Tel: +1-507-284-2174, Fax: +1-507-255-7612, E-mail: todd@mayo.edu
}

DOI: $10.5009 / \mathrm{gnl} .2010 .4 . \mathrm{S} 1 . \mathrm{S} 50$ 
classification system for the three anatomic scenarios of duodenal obstruction in relation to the major papilla that determine the endoscopic approach and technical success to combined palliation of biliary and duodenal obstruction. The classification system is as follows (Fig. 1):

Type I stenosis occurs at the level of the duodenal bulb or upper duodenal genu but without involvement of the papilla. Type II stenosis affects the second part of the duodenum with involvement of the major papilla. Type III stenosis involves the third part of the duodenum distal to and without involvement of the major papilla.

Of the three types of biliary-duodenal stenoses, technical difficulty to achieve successful combined biliary and

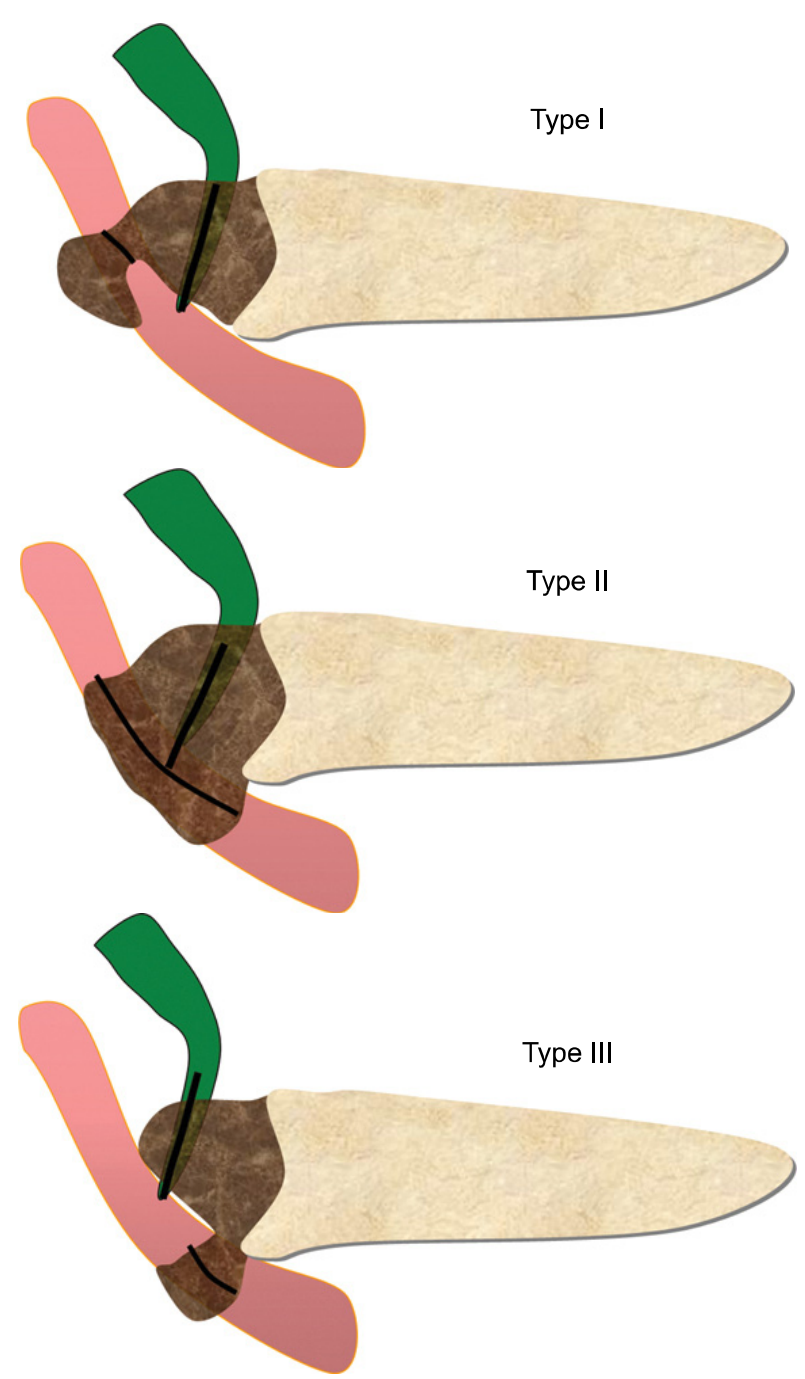

Fig. 1. Classification of duodenal stenosis type in relation to the major papilla as proposed by Mutignani et al. Endoscopy 2007;39:440-447. ${ }^{1}$ : (I) at the level of the duodenum proximal to and without involvement of the papilla, (II) affecting the second part of the duodenum with involvement of the major papilla, and (III) involving the third part of the duodenum distal to and without involvement of the major papilla. duodenal palliation is least in patients with type III duodenal stenosis while type I is intermediate and type II is the most technically difficult.

The approach to type I cases is to pass the duodenoscope through the duodenal stricture to the major papilla, if possible. This may require balloon dilation of the stricture to a diameter of 15-18 mm (Fig. 2A) and/or passing the balloon to the third duodenum to be used as an anchor to pull the endoscope across the stricture. ${ }^{5}$ Once the major papilla is reached the biliary tree is cannulated and an expandable metal biliary stent is placed (Fig. 2B). A guidewire is then advanced through the channel of the endoscope and passed into the fourth portion of the duodenum; the duodenoscope is withdrawn into the stomach and a duodenal stent which passes through the working channel of the endoscope is deployed across the duodenal stricture as previously described (Fig. 2C). ${ }^{6}$ It is usually necessary to place the proximal end of the stent into the stomach to allow enough stent coverage of the duodenal stricture since type I strictures tend to be in the proximal duodenal bulb. If the duodenoscope cannot be advanced through the stricture despite balloon dilation a duodenal stent is placed across the stricture. Since the luminal diameter of commercially available duodenal stents is 20-22 $\mathrm{mm}$ and duodenoscopes are approximately $11 \mathrm{~mm}$, the endoscope can usually be passed through the stent during the same procedure to allow the biliary system to be accessed with placement of a biliary SEMS. However, this may require balloon dilation of the duodenal stent. It is important that the duodenal stent be placed with the distal end positioned proximal to the level of the major papilla to allow the bile duct to be accessed (Fig. 3). The location of the major papilla can be estimated easily fluoroscopically in the presence of a prior biliary stent or by passing a smaller caliber forward-viewing endoscope to the papilla and a obtaining a radiographic image with the endoscope positioned at the level of the papilla for later reference. If the duodenoscope cannot be passed through the duodenal stent lumen because of inadequate stent expansion despite balloon dilation there are three options. The first option is to repeat the endoscopic retrograde cholangiopancreatography (ERCP) after waiting at least 48 to 72 hours at which time the duodenal stent is almost always fully expanded and will allow passage of the duodenoscope to the second duodenum. Other options to palliate biliary obstruction include percutaneous access to the biliary tree and endoscopic ultrasound (EUS)-guided entry into the biliary tree (both of these approached will be discussed in more detail below under management of type II duodenal obstruction).

The approach to type II cases is most difficult. The sec- 


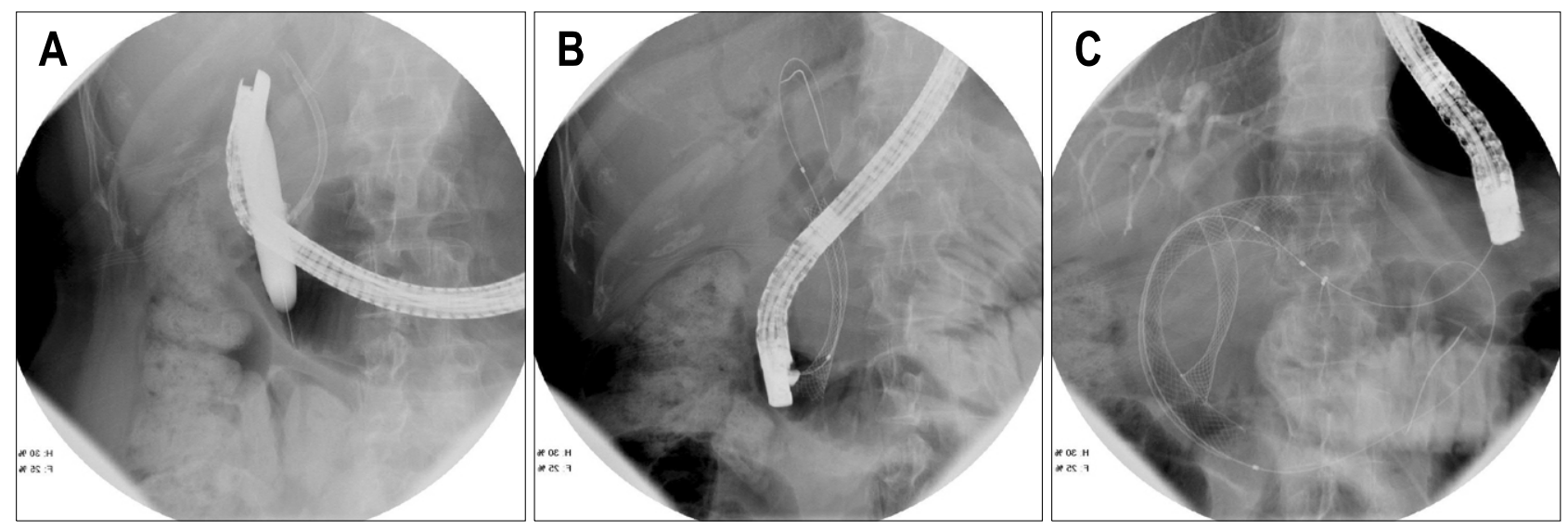

Fig. 2. Palliation of biliary and duodenal obstruction in type I stenosis. (A) The duodenal stricture is balloon dilated to allow passage of the duodenoscope to the level of the major papilla (note the prior placement of a plastic biliary stent). (B) After the endoscope is passed to the major papilla, the plastic stent is removed and an expandable metal biliary stent is deployed. (C) After deployment of the biliary stent, an expandable metal duodenal stent is placed.
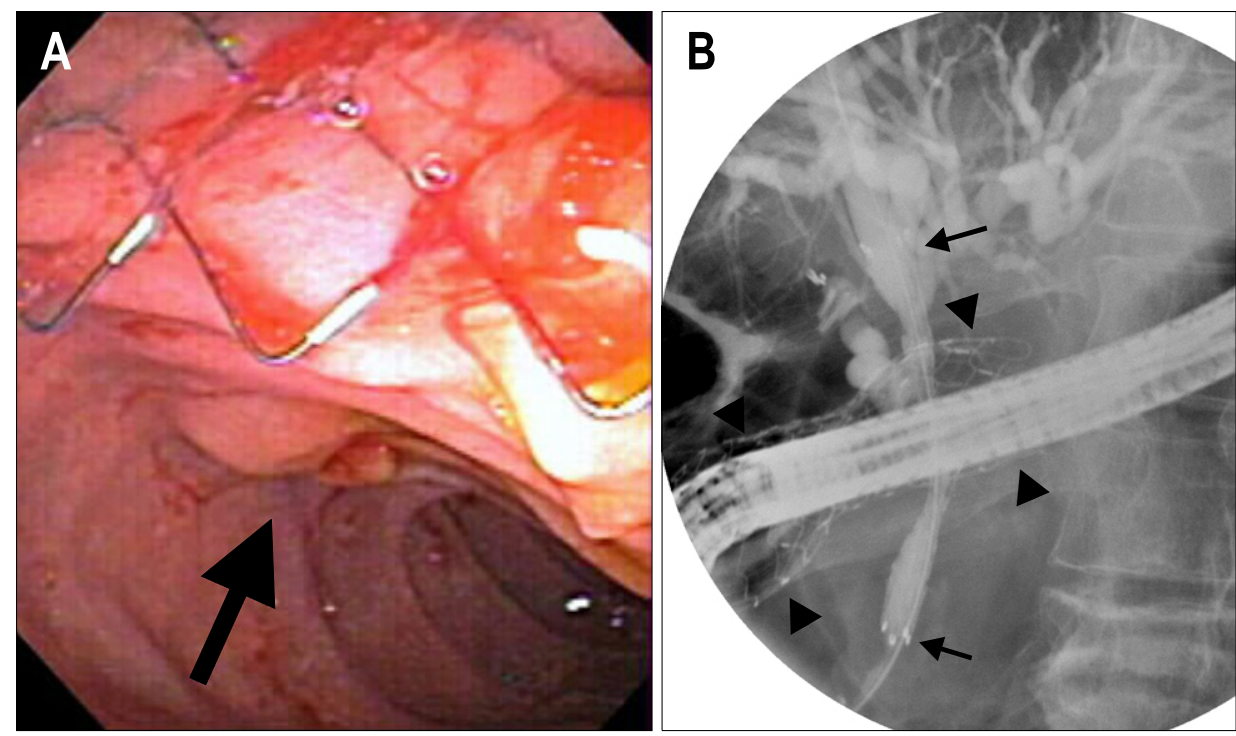

Fig. 3. Type I duodenal stenosis with duodenal stent placement to allow access to the bile duct. (A) The duodenal stent is positioned with the distal end proximal to the major papilla (arrow) as visualized using an upper endoscope. (B) In the same patient, the endoscope has been passed through the duodenal stent (arrowheads) lumen and an expandable metal biliary stent (arrows) is placed.

ond duodenum is strictured and involves the major papilla. Unless the patient has had a prior transpapillary biliary stent placed, bile duct cannulation is often not successful since the major papilla is not endoscopically identifiable due to extensive tumor infiltration. In addition, the lumen is narrowed and there is little working room between the lens of the endoscope and the major papilla. Nonetheless, one should first attempt placement of an expandable metal biliary stent. If successful, the duodenal stent is then placed across the stricture and overlies the biliary stent. If identification and/or cannulation of the major papilla cannot be achieved then a duodenal stent is placed across the stricture. Unfortunately, the stent will invariably further impair endoscopic visualization of the major papilla. In some cases, however, the major papilla can be identified through the interstices of the duodenal stent and the bile duct accessed with placement of a biliary SEMS. If the bile duct cannot be accessed through a transpapillary approach after duodenal stent placement then biliary access can be achieved using a percutaneous or EUS approach. ${ }^{7}$ Typically, in either of the two approaches the bile duct is accessed through the liver (when EUS is used a transgastric approach is most often used). In addition, in either approach there are two options: a rendezvous approach or completion alone using a percutaneous or EUS approach. When the rendezvous approach is used, a guidewire is passed into the biliary tree across the stricture and through the interstices of the stent into the stent lumen (Fig. 4A, B); if the EUS approach is used, the echoendoscope is removed. A duode- 

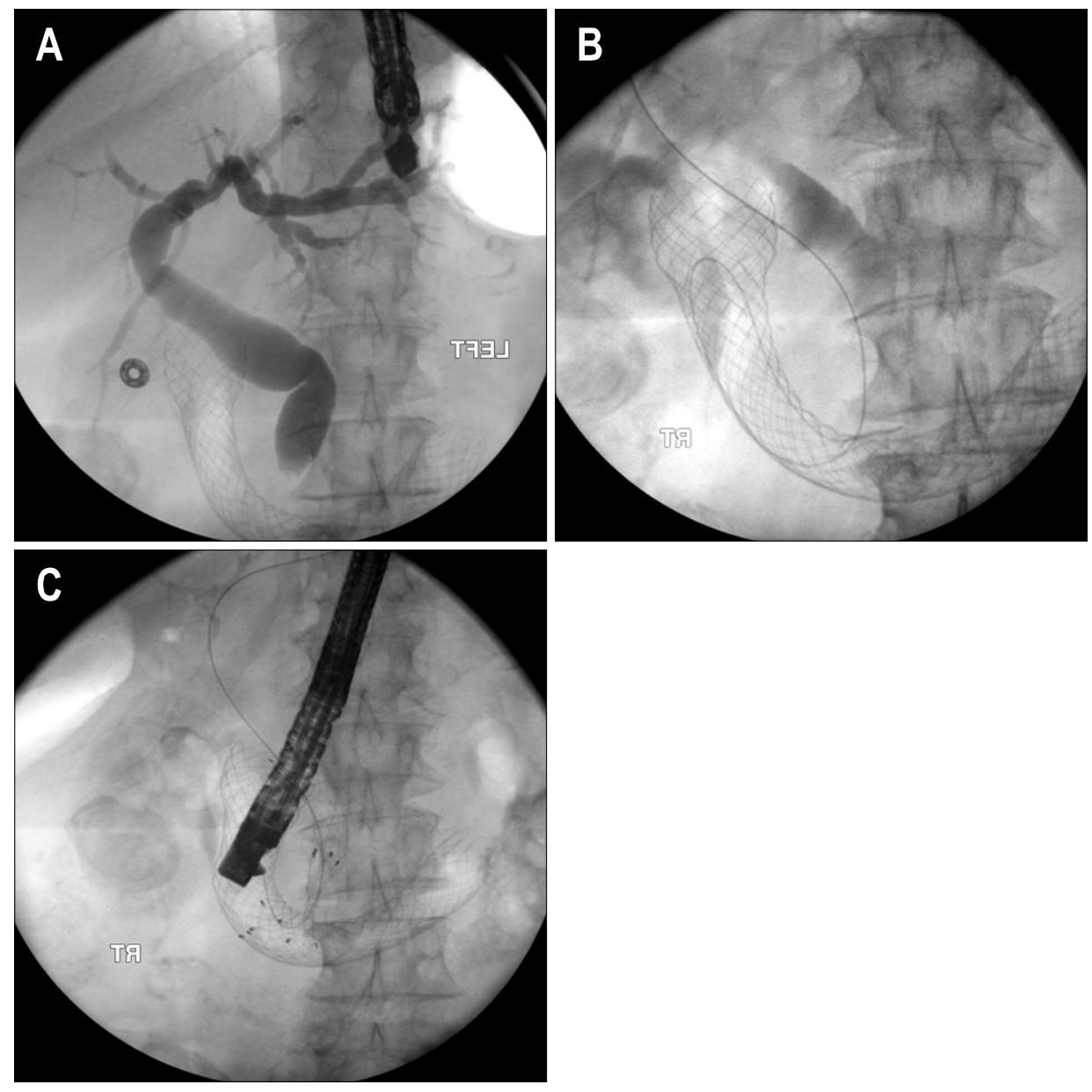

Fig. 4. Successful simultaneous biliary and duodenal stent placement using the endoscopic ultrasound (EUS) rendezvous technique in type II duodenal stenosis. (A) After placement of a duodenal self-expandable metal stent (SEMS), the bile duct could not be cannulated. A transgastric EUS-guided puncture was performed. (B) A guide wire was passed into the lumen of the duodenal stent. (C) After the echoendoscope was withdrawn, a duodenoscope was advanced into the duodenal stent lumen, the guide wire grasped, and a biliary SEMS deployed.

noscope is advanced into the lumen of the duodenal stent and the guidewire wire is grasped with a snare and withdrawn into the channel of the endoscope. A biliary stent advanced through the endoscope channel over the guidewire, into the biliary tree and deployed. The distal end of the biliary stent resides within the lumen of the duodenal stent (Fig. 4C). When the biliary stent is placed entirely using the percutaneous approach, the stent is passed antegrade and the distal end is positioned into the duodenal lumen as described above (Fig. 5). When the EUS approach is undertaken, the distal end of the biliary stent can reside within the biliary tree proximal to the biliary stricture and with the proximal end deployed into the gastric lumen to create a hepaticogastric anastomosis. However, this approach is associated with a higher rate of bile leakage than when the stent crosses the papilla and into the duodenum. The latter EUS-guided approach is similar to the percutaneous approach in which the guidewire is passed antegrade into the duodenum across the duodenal stricture. The stent is then passed through the echoendoscope and deployed across the stricture with the distal end into the duodenum. ${ }^{8}$ Finally, in a recent case report an EUS-guided approach was performed where the echoendoscope was passed into the lumen of a previously placed duodenal stent in a type II stenosis in which the papilla could not otherwise be endoscopically visualized. The bile duct opening was identified and a transpapillary biliary SEMS placed. ${ }^{9}$

Type III cases are the least common and often result from pancreatic cancer that arises from the uncinate process. The tumor encases the bile duct causing biliary obstruction and extends inferiorly to causing duodenal 


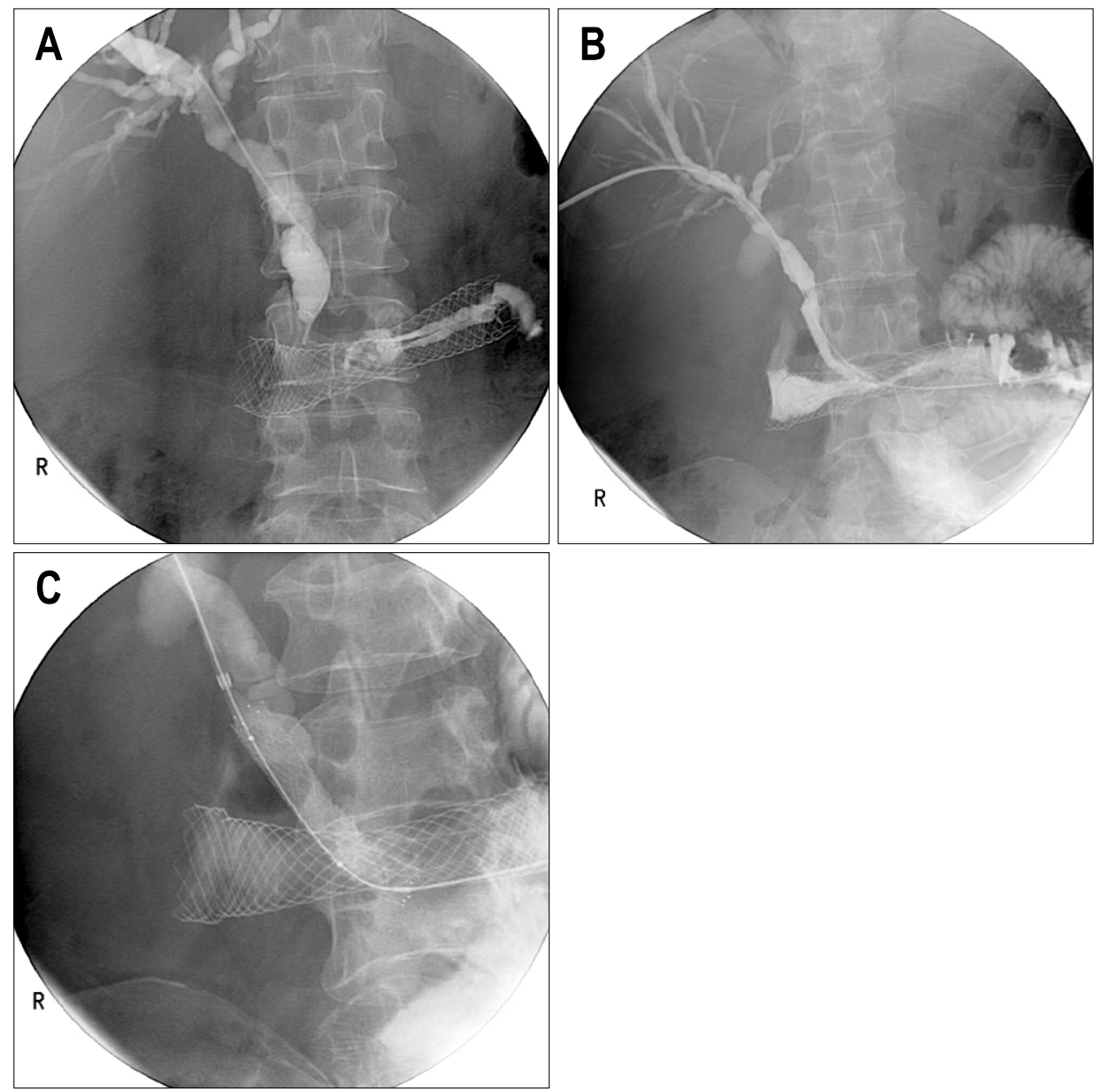

Fig. 5. Successful combined biliary and duodenal stent placement using a percutaneous approach in type II duodenal stenosis. A duodenal stent was endoscopically placed and tumor-precluding endoscopic bile-duct cannulation was performed in the duodenum. (A) Percutaneous cholangiography demonstrates a tight distal bile-duct stricture. (B) A guide wire was passed into the lumen of the duodenal stent. (C) After balloon dilation of the stent interstices, a biliary self-expandable metal stent (SEMS) was deployed percutaneously as a one-step procedure.
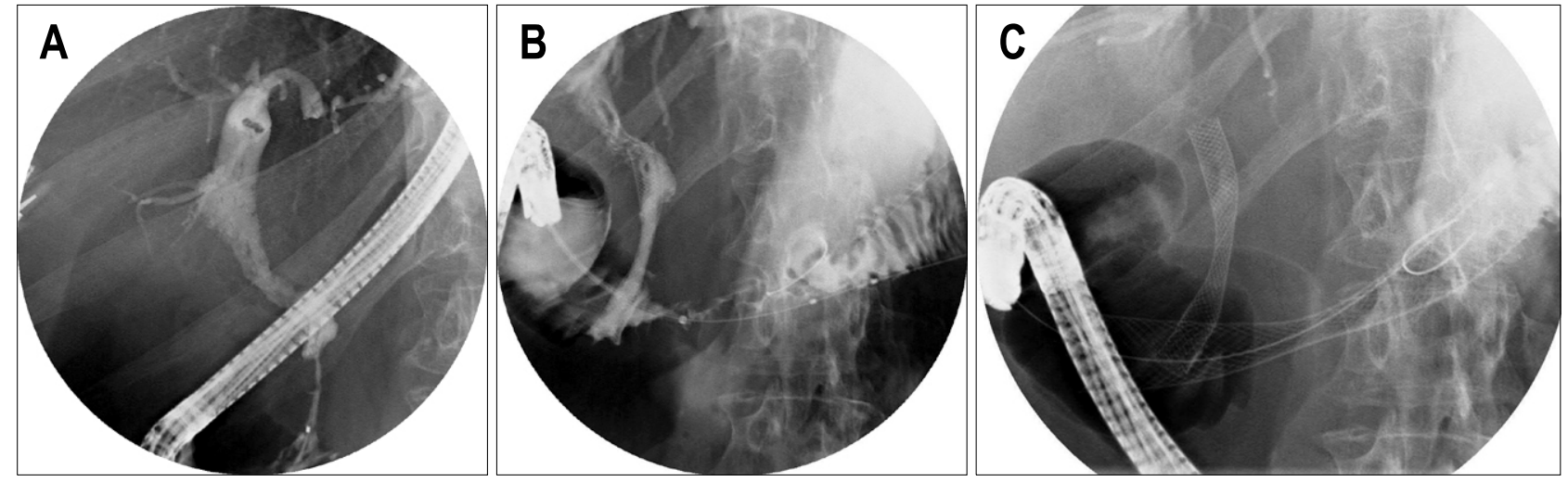

Fig. 6. Successful combined endoscopic biliary and duodenal stent placement in type III duodenal stenosis. (A) The bile duct with a distal stricture is cannulated. (B) After placement of the biliary self-expandable metal stent (SEMS), the duodenal stricture is delineated with contrast agent. (C) The duodenal SEMS is deployed across the duodenal stricture with the proximal end just distal to the biliary SEMS. 
obstruction below the level of the major papilla. These cases are the least technically difficult since the duodenoscope can be passed to the major papilla and to the level of the stricture. In addition, it is not necessary to pass the endoscope beyond the duodenal stricture and thus balloon dilation of the stricture is not necessary. The sequence of SEMS placement (biliary stent first then duodenal stent or vice versa) is usually not critical when the distance between the major papilla and the proximal portion of the biliary stricture are not in close proximity (Fig. 6). However, if the proximal level of the duodenal obstruction is very close to the major papilla it is best to place the biliary stent first since the proximal end of the duodenal stent may need to be placed across the level of the major papilla to allow adequate coverage of the duodenal stricture. Whatever sequence of stent placement is chosen, it is best to avoid placing the duodenal stent across the biliary opening so that biliary access is preserved both at the time of the initial procedure as well as in the future should biliary stent occlusion occur.

\section{Clinical scenarios}

In addition to the relationship of the duodenal obstruction to the major papilla other factors influence the endoscopic approach. These include prior surgical palliation (gastrojejunal bypass) and clinical scenarios. The most common clinical scenario is the initial development of biliary obstruction followed by later onset of duodenal obstruction. Many patients with duodenal obstruction have already undergone a palliative intervention for relief of biliary obstruction - endoscopic, percutaneous, or surgical. If a transpapillary stent was previously placed then the type and timing of prior stent placement needs to be determined. For example, if a plastic biliary stent had been previously placed it is likely occluded or will become occluded and needs to be replaced. In such patients it is recommended that a metal biliary stent be placed at the time of duodenal stent placement, especially in type II patients (Fig. 2) since the duodenal stent must cross the level of the major papilla making subsequent biliary stent placement difficult, if not impossible since it may not be accessible through the interstices of the duodenal stent.

Another scenario is simultaneous presentation of gastric and duodenal obstruction without prior intervention. In this scenario it is recommended that placement of a biliary SEMS be performed during the same procedure as placement of the duodenal SEMS, if possible.

An additional clinical scenario is duodenal obstruction followed by later biliary obstruction. This is uncommon and could be difficult to treat endoscopically since the papilla is usually inaccessible if the duodenal stent was

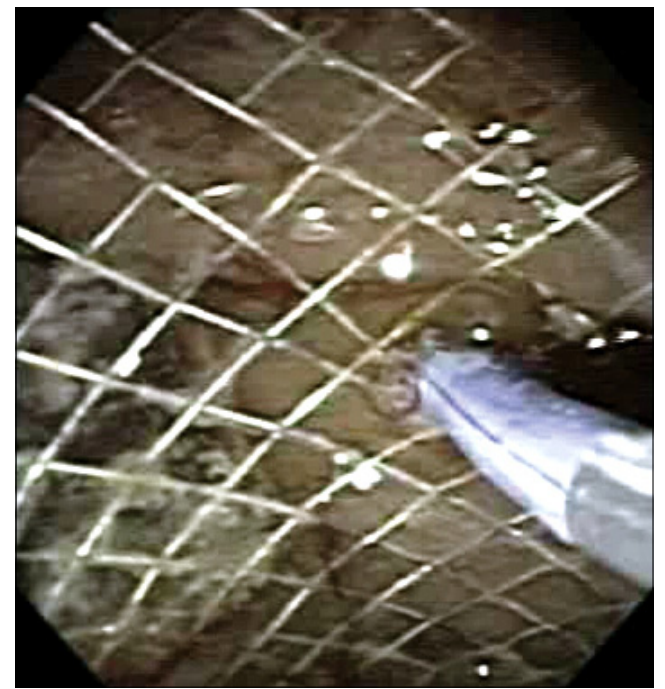

Fig. 7. Endoscopic photograph demonstrates successful cannulation of the bile duct in a type I patient in whom a gastroduodenal self-expandable metal stent (SEMS) was placed across the papilla 3 days earlier. A biliary SEMS was placed.

placed across the papilla. If the stent does not cross the papilla, the endoscope can be passed through the metal stent and ERCP performed as usual. If the stent has crossed the papilla in a type I duodenal stenosis, it may be possible to identify a normal papilla through the interstices of the stent followed by successful cannulation of the bile duct (Fig. 7) or to create a window within the stent at the level of the papilla using rat-toothed forceps or argon plasma coagulation. ${ }^{1,10}$ If these maneuvers fail, then either a percutaneous or EUS-guided approach to placement of a metal biliary stent is usually required, as described above.

Finally, when considering endoscopic management of duodenal obstruction in the patient without clinically overt biliary obstruction, prophylactic placement of a biliary SEMS should be considered, especially if there is any evidence of biliary ductal dilation by non-invasive imaging or in the presence of abnormal liver function tests that cannot be explained by other processes such as medications or presence of liver metastases.

\section{Results}

There are several series describing successful combined biliary and endoscopic drainage. ${ }^{1,11-13}$ In an early study ${ }^{11}$ 18 patients underwent simultaneous biliary and duodenal self-expandable metal stent. Ten patients had prior plastic biliary stents in place. Combined metal stenting was technically successful in 17 patients. All the patients had relief of biliary obstruction and 16 had a relief of gastric 
outlet obstructive symptoms. No immediate stent-related complications occurred. Median survival time was 78 days. The authors who devised the duodenal stenosis classification (Fig. 1) ${ }^{1}$ achieved technically successful combined biliary and duodenal stent placement in the vast majority of patients in all three types of duodenal stenosis, though the majority of patients (46/64) had biliary stents placed at a mean interval of 107 days prior to duodenal stent placement; prior biliary stent placement greatly facilitates successful combined stenting in type II duodenal stenoses. However, some of these patients required rendezvous procedures. Early complications occurred in $6 \%$ of patients and late complications occurred in $16 \%$. The median survival after combined stenting was 81 days. In another series 23 patients who had both biliary and duodenal stenoses, successful combined stenting was achieved in $91 \%$ of cases. ${ }^{12}$ More recently, the use of a dedicated duodenal stent with a central portion designed to facilitate passage of a biliary stent through the interstices was reported in a small number of patients. ${ }^{13}$ Endoscopic placement of duodenal SEMS was achieved in all and a self-expandable metal biliary stent through the mesh of the duodenal stent was technically successful in $7(87.5 \%)$ of 8 patients. However, $2 / 3$ patients with type II duodenal strictures failed bile duct cannulation and required a rendezvous procedure. Early complications occurred in 1 patient. Median survival after combined stenting was 91 days (range, 36-314 days).

In summary, the results of these series suggest that in experienced centers combined biliary and duodenal stent placement for palliation can be achieved in the majority of patients though a rendezvous approach is required more often in the type II patients. Overall survival from the time of combined biliary and duodenal stent placement is relatively short.

\section{REFERENCES}

1. Mutignani M, Tringali A, Shah SG, et al. Combined endoscopic stent insertion in malignant biliary and duodenal obstruction. Endoscopy 2007;39:440-447.
2. Moss AC, Morris E, Leyden J, MacMathuna P. Do the benefits of metal stents justify the costs? A systematic review and meta-analysis of trials comparing endoscopic stents for malignant biliary obstruction. Eur J Gastroenterol Hepatol 2007; 19:1119-1124.

3. Jeurnink SM, Steyerberg EW, van Hooft JE, et al. Surgical gastrojejunostomy or endoscopic stent placement for the palliation of malignant gastric outlet obstruction (SUSTENT study): a multicenter randomized trial. Gastrointest Endosc 2010;71:490-499.

4. Adler DG, Baron TH. Endoscopic palliation of malignant gastric outlet obstruction using self-expanding metal stents: experience in 36 patients. Am J Gastroenterol 2002;97: 72-78.

5. Kikuyama M, Itoi T, Sasada Y, Sofuni A, Ota Y, Itokawa F. Large-balloon technique for one-step endoscopic biliary stenting in patients with an inaccessible major papilla owing to difficult duodenal stricture (with video). Gastrointest Endosc 2009;70:568-572.

6. Baron TH. Optimizing endoscopic placement of expandable stents throughout the GI tract. Expert Rev Gastroenterol Hepatol 2008;2:399-409.

7. Shami VM, Kahaleh M. Endoscopic ultrasound-guided cholangiopancreatography and rendezvous techniques. Dig Liver Dis 2010;42:419-424.

8. Nguyen-Tang T, Binmoeller KF, Sanchez-Yague A, Shah JN. Endoscopic ultrasound (EUS)-guided transhepatic anterograde self-expandable metal stent (SEMS) placement across malignant biliary obstruction. Endoscopy 2010;42: 232-236.

9. Belletrutti PJ, Gerdes H, Schattner MA. Successful endoscopic ultrasound-guided transduodenal biliary drainage through a pre-existing duodenal stent. JOP 2010;11:234236.

10. Topazian M, Baron TH. Endoscopic fenestration of duodenal stents using argon plasma to facilitate ERCP. Gastrointest Endosc 2009;69:166-169.

11. Kaw M, Singh S, Gagneja H. Clinical outcome of simultaneous self-expandable metal stents for palliation of malignant biliary and duodenal obstruction. Surg Endosc 2003; 17:457-461.

12. Maire F, Hammel P, Ponsot P, et al. Long-term outcome of biliary and duodenal stents in palliative treatment of patients with unresectable adenocarcinoma of the head of pancreas. Am J Gastroenterol 2006;101:735-742.

13. Moon JH, Choi HJ, Ko BM, et al. Combined endoscopic stent-in-stent placement for malignant biliary and duodenal obstruction by using a new duodenal metal stent (with videos). Gastrointest Endosc 2009;70:772-777. 